\title{
Automatic Test Bench for Measurement of Magnetic Interference on LVDTs
}

\author{
Giovanni Spiezia, Roberto Losito, Michele Martino, Alessandro Masi, and Antonio Pierno
}

\begin{abstract}
This paper proposes an automatic test bench, based on a rigorous and repeatable measurement procedure, for the analysis of a position sensor linear variable differential transformer (LVDT). The test bench allows complete characterization of an LVDT sensor working in an interfering magnetic field by evaluating the uncertainty and nonlinearity of the sensor. This issue is addressed in neither the sensor datasheet nor the scientific literature. The potential of the method and the performance of the automated test bench are proven by measuring the main sensor characteristics such as nonlinearity and uncertainty, and the error of a position reading due to external magnetic interference on two commercial LVDTs. The sensors are based on two different reading techniques, and both are analyzed using current and voltage excitations. In fact, the test bench permits to study the robustness of the sensor with respect to external magnetic fields by comparing position drifts due to interference at varying source excitations and reading techniques.
\end{abstract}

Index Terms-Automatic testing, magnetic field effects, magnetic field measurements, magnetic variable measurements.

\section{INTRODUCTION}

A UTOMATIC test benches are more and more used to reduce the duration of a measurement procedure to characterize a given product. In addition to time advantage, a test bench improves repeatability of results, assuring better quality. For these reasons, it can become a reference tool for the characterization and evaluation of the performance figures of a given product. In order to achieve that, the test bench must be based on a valid method and procedure, which addresses the main features of a device under test. This paper wants to propose a test bench and a measurement procedure for the characterization of a linear variable differential transformer (LVDT) in the presence of an external magnetic field [1]. In the following, the basic principles of the LVDT are described, and then, the reasons to develop an automatic test bench are given.

Manuscript received June 24, 2010; revised September 28, 2010; accepted December 12, 2010. Date of publication February 24, 2011; date of current version April 6, 2011. The Associate Editor coordinating the review process for this paper was Dr. Subhas Mukhopadhyay.

G. Spiezia, R. Losito, and A. Masi are with the European Organization for Nuclear Research, CH-1211 Geneva, Switzerland (e-mail: Giovanni. Spiezia@cern.ch; Roberto.Losito@cern.ch; Alessandro.Masi@cern.ch).

M. Martino and A. Pierno are with the European Organization for Nuclear Research, CH-1211 Geneva, Switzerland, and also with the University of Naples, 80125 Naples, Italy (e-mail: Michele.Martino@cern.ch; Antonio.Pierno@cern.ch).

Color versions of one or more of the figures in this paper are available online at http://ieeexplore.iee.org.

Digital Object Identifier 10.1109/TIM.2011.2112990

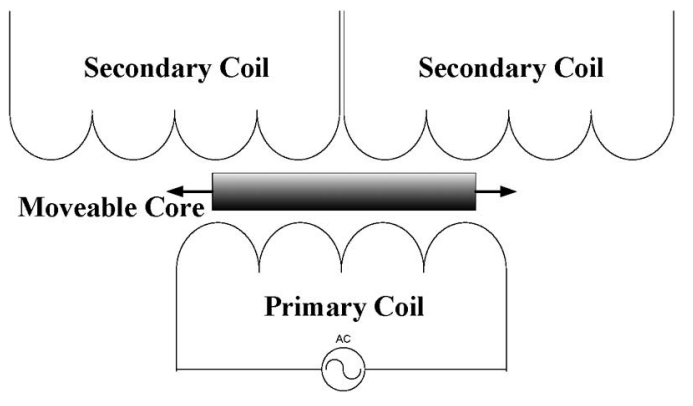

Fig. 1. LVDT schematic.

\section{A. LVDT Working Principle}

An LVDT is a magnetic displacement sensor that allows accurate measurement of position. The LVDT is one of the most widespread positioning sensors used in harsh environments such as industrial plants, nuclear plants, and particle accelerators, due to contactless sensing, good linearity, virtually infinite resolution, low temperature sensitivity, robustness, and easy-to-implement radiation hardness. Therefore, the LVDT is used in a large variety of applications such as in the industry and in research centers [2]-[6]. It is a differential transformer consisting of one primary and two secondary coils, which are wound around a metallic foil. A moveable part, i.e., the core, which is made of a high-magnetic-permeability material, is inserted in the foil. A sine waveform is applied to the primary coil to excite the magnetic circuit, generating on the secondary coils a sine wave whose amplitude depends on the core position (see Fig. 1).

Theoretically, the LVDT sensor has no limit in terms of resolution, which is then determined by its transducer, i.e., the conditioning and acquisition system of the primary and secondary coils. In fact, several methods exist to evaluate the position on the basis of the amplitude of the secondary coils, which are basically amplitude demodulation procedures [7]. The reading technique also depends on LVDT type and, more specifically, on the available wires of the coils. A four-wire LVDT provides two wires for the primary coil and two wires for the secondary coils, which are connected internally in phase opposition. A five-wire model makes available the central point of the series of the secondary coils, whereas a six-wire model provides all the output legs of the coils. Different techniques have been proposed and investigated [3], [7]-[10]. The three main techniques are differential reading $\left(V_{\mathrm{sec} 1}-V_{\mathrm{sec} 2}\right)$, normalized differential reading $\left(V_{\mathrm{sec} 1}-V_{\mathrm{sec} 2}\right) / V_{\mathrm{pri}}$, and ratiometric reading $\left(V_{\mathrm{sec} 1}-V_{\mathrm{sec} 2}\right) /\left(V_{\mathrm{sec} 1}+V_{\mathrm{sec} 2}\right)$. $V_{\mathrm{sec} 1}, V_{\mathrm{sec} 2}$, and $V_{\text {pri }}$ are the amplitudes of two secondary voltages and a primary voltage, respectively. 
The digital technique based on ratiometric function, which links the core position to the ratio between the difference and the sum of the output voltages of the secondary coils, is mostly used for a five- or a six-wire model since it assures high performance, and the reading position is independent of the primary coil variation [7].

\section{B. Magnetic Interference on the LVDT}

Although the LVDT is one of the most widespread positioning sensors used in harsh environments, its accuracy is strongly affected and reduced by slowly varying external magnetic fields impinging on the LVDT structure. Manufacturers are aware of the effects of external magnetic fields, and some of them state that an internal shield is provided. However, the specifications on the maximum tolerable magnetic field and the consequent accuracy reduction are not detailed. The effects on the LVDT reading position can depend on external interference features such as its intensity and direction, as well as on sensor features, such as its materials and structure [1], [11]. Although such interference, due to power supply cables or stray fields of equipment installed close to the sensor, affects deeply the correct working of the sensor, this issue is not well addressed in the literature [1], [11].

\section{Proposal}

A well-defined procedure is given for measuring the output characteristic of the sensor. According to its design and its available wires, a differential or a ratiometric reading [3], [7][10] is used, and the nonlinearity and uncertainty of the sensor are given. Then, the procedure is detailed to analyze the sensor under test in the presence of an external magnetic field on the LVDT. The method aims at evaluating the accuracy reduction of an LVDT due to magnetic interference. The procedure is proposed as a reference to test the LVDT sensors in the presence of an external magnetic field. An automatic test bench implements this procedure and has been improved with respect to that in [12] in order to be exploited as a tool to find an optimal supplying excitation and a reading technique under which sensitivity of a given sensor to interference is minimized. In Section II, the measurement conditions and the method are specified; in Section III, the test bench is described. Finally, experimental results, carried out on two commercial LVDTs to qualify the test bench, are shown and discussed in Section IV.

\section{Measurement Conditions And Methods}

Measurement conditions and procedures have to be defined in order to fix a suitable method for different types of LVDT sensors. As far as the reading of the sensor is concerned, the primary coil is fed by a voltage or a current sine waveform at a frequency ranging from 1 to $5 \mathrm{kHz}$. Although a few LVDTs are designed for operation at $60 \mathrm{~Hz}$, excitation frequencies of $250 \mathrm{~Hz}$ to $10 \mathrm{kHz}$ are more typical, with $1 \mathrm{kHz}$ being the most common. Generally, a higher frequency is desired in order to have a faster response of the LVDT to variation in the position. A limiting factor, however, is that an excessively high frequency
$(>10 \mathrm{kHz}$ ) leads to eddy current loss in the core and results in lower output signal level, more power dissipation, and greater temperature sensitivity [13].

The amplitude value is chosen in order to have some units of volts on the secondary coils. A high-resolution high-samplingrate data acquisition board is used to acquire the primary and both secondary waveforms, whose amplitudes are evaluated by applying a sine-fit algorithm on 2000 samples acquired at $250 \mathrm{kS} / \mathrm{s}$.

Then, the ratiometric reading function is applied, i.e., the difference of the secondary voltage amplitudes over its sum $\left(\left(V_{\sec 1}-V_{\sec 2}\right) /\left(V_{\sec 1}+V_{\sec 2}\right)\right)$. In case of a four-wire model, only the antiphase series of the secondary coils is available; therefore, the reading technique suggested by the manufacturer is exploited [see Fig. 2(a)].

The sensor is first calibrated without any interference magnetic field [see Fig. 2(a)]. Let us assume that the sensor works in the position range $(-P,+P)$. Moving the core from position $-P$ to $+P$, a conversion table $T$ is created between the array of position readings $p_{i}$ and their corresponding ratiometric values $r_{i}$. The reference positions $p_{i}$ are measured by another independent displacement sensor. At each position $p_{i},(N=50)$ repeated measurements are done to evaluate uncertainty $u_{i}$ [see Fig. 2(a)]. Then, the average of $u_{i}$ is evaluated. It represents the uncertainty $u_{\mathrm{LVDT}}+\mathrm{read}$ of the LVDT sensor and the associated reading system. As a matter of fact, the uncertainty of the sensor itself cannot be evaluated without the reading system; in fact, the reading system can be regarded as the transducer of the sensor. At this stage, nonlinearity NL is evaluated as well [see Fig. 2(a)].

This procedure is then repeated $(M=30)$ times to evaluate the uncertainty of the overall bench. In fact, moving the core $M$ times between positions $-P$ and $+P$ allows evaluating uncertainty $u_{p}$ of the positioning system by means of the external reference sensor. Evaluation of this figure is significant to compare more sensors and assess the effect of the external magnetic field. This point will be detailed in Section IV. Finally, composition of $u_{p}$ and $u_{\mathrm{LVDT}}+\mathrm{read}$ gives the overall uncertainty $u$ of the test bench and of the LVDT under test [see Fig. 2(a)].

The described procedure is then applied in the case of an external magnetic source. A new set of ratiometric values $r_{i}^{\prime}$ is measured and then converted in the position reading $p_{i}^{\prime}$ using the calibration table $T$ [see Fig. 2(b)]. The difference $p_{i}-p_{i}^{\prime}$, where $p_{i}$ is the reference position read by another sensor, is the position drift or position error due to magnetic interference and chosen as figure of merit to evaluate the performance of the LVDT sensors in the presence of the external magnetic field. In this paper, the position drift will be measured for two commercial LVDTs.

A transversal or a longitudinal magnetic field impacting uniformly on the LVDT sensor structure is considered as the external source [see Fig. 2(b)]. They are separately applied, assuming that the effect of a field, impinging at any angle, can be always considered as the contribution of its transversal and axial components [1]. In particular, the transversal field is generated by means of a resistive magnet and the longitudinal one by means of a solenoid. 


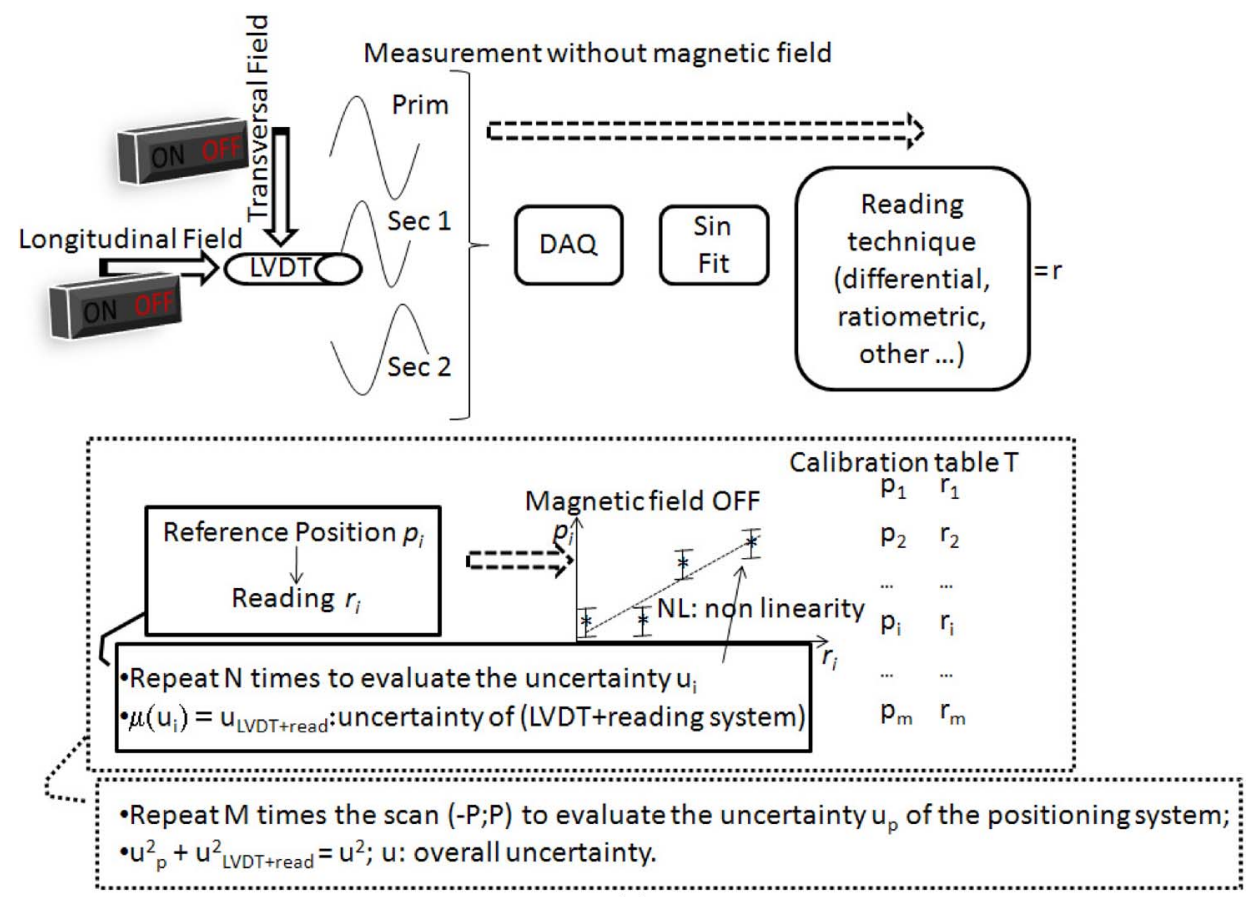

(a)

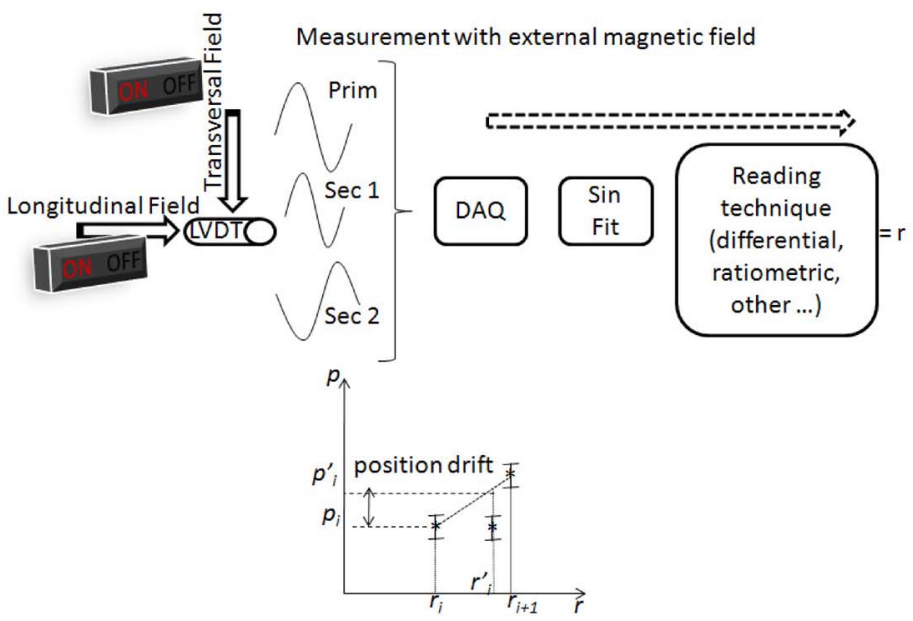

(b)

Fig. 2. (a) Chart of the measurement procedure. The calibration table $T$, nonlinearity, uncertainty of the LVDT and the reading system, uncertainty of the positioning system, and overall uncertainty are evaluated for measurement without any magnetic interference. (b) Position drift is evaluated for the measurement with a magnetic field impinging on the LVDT structure.

The evaluation of the position drift is carried out as follows: 1) For a given position $p_{i}$ and a maximum external field $|H|$, a first cycle from 0 to $|H|$ and then to $-|H|$ and back to $|H|$ is applied with no measurements. 2) An interfering magnetic field scan is performed by following the previous cycle, changing the field with step $\Delta H$. For each magnetic field value, the ratiometric value $r_{i}^{\prime}$ is measured (over $N=50$ repeated measurements). 3) A demagnetization procedure is performed. 4) The core is moved to position $p_{i+1}$.

The demagnetization procedure resulted to be a critical point of the measurement procedure. It is necessary to bring the core and the foil back to their initial magnetic state, before starting a measurement at a new position. If those magnetic parts of the LVDT are not correctly demagnetized, repeatability of the measurement conditions is not assured. Therefore, a demagnetization procedure is applied for each measurement with an external field of intensity $|H|$ at a given position $p_{i}$. Starting from the field value $|H|$, repeated magnetic cycles, with decreasing amplitude, are applied to demagnetize the sensor materials. The shape of the decreasing amplitude is depicted in Fig. 3. A linear shape was preferred to the exponential one chosen at the first stage [12] since the former assures a smoother decrease in the magnetic field than exponential decay, above all at high values of $H$. This allowed improving the effectiveness of LVDT material demagnetization.

The value of the position reading before applying the external magnetic field $|H|$ and that after the measurement procedure and the demagnetization cycle are compared. If the difference 


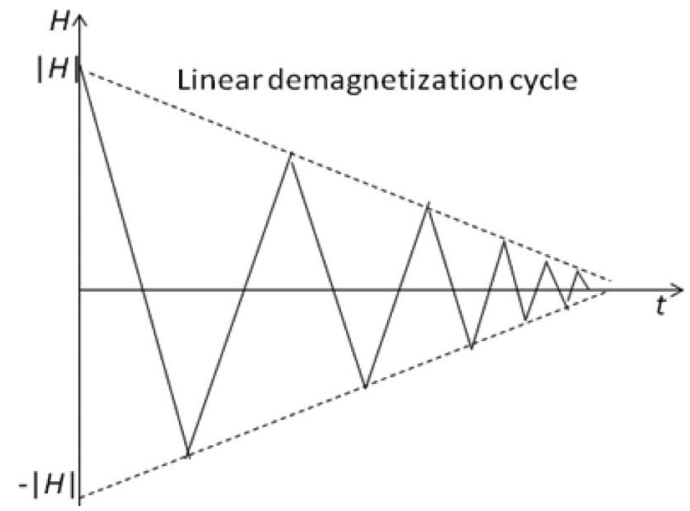

Fig. 3. Amplitude shape of the applied magnetic field for the demagnetization cycle.

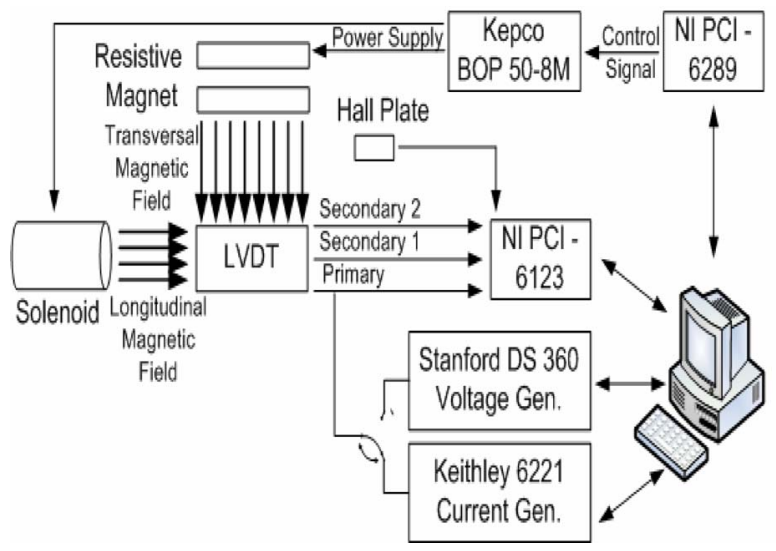

Fig. 4. LVDT test bench.

between the aforementioned values is within the sensor's uncertainty, then the demagnetization procedure succeeded; otherwise, it is repeated.

\section{TEST BENCH}

The test bench is depicted in Fig. 4. The resistive magnet SIGMAPHI F12407 and the solenoid, which are used as transversal and longitudinal magnetic interference sources, respectively, are driven by the KEPKO BOP 50-8M amplifier whose control voltage is generated by means of an analog output of the data acquisition board NI-PCI 6289. The primary coil is connected to the voltage generator Stanford DS 360 or to the current generator Keithley 6221 according to whether one wants to drive the sensor in the voltage or the current mode. The primary and both secondary coils are acquired by means of the data acquisition board NI-PCI 6123. A step motor is used to move the LVDT core, and the absolute reference position is measured by means of the photoelectric linear encoder Heidenhain LIP 401R, whose uncertainty is $0.5 \mu \mathrm{m}$. The test bench is remotely controlled by using a software program developed in LabView.

\section{A. Magnetic Sources}

The external field, which was generated by the solenoid or by the resistive magnet, has been measured by means of a Hall plate Senis $\mathrm{GmbH} 3 \mathrm{MH} 3 \mathrm{C}-\mathrm{H} 3 \mathrm{~A}$ to find out the transfer

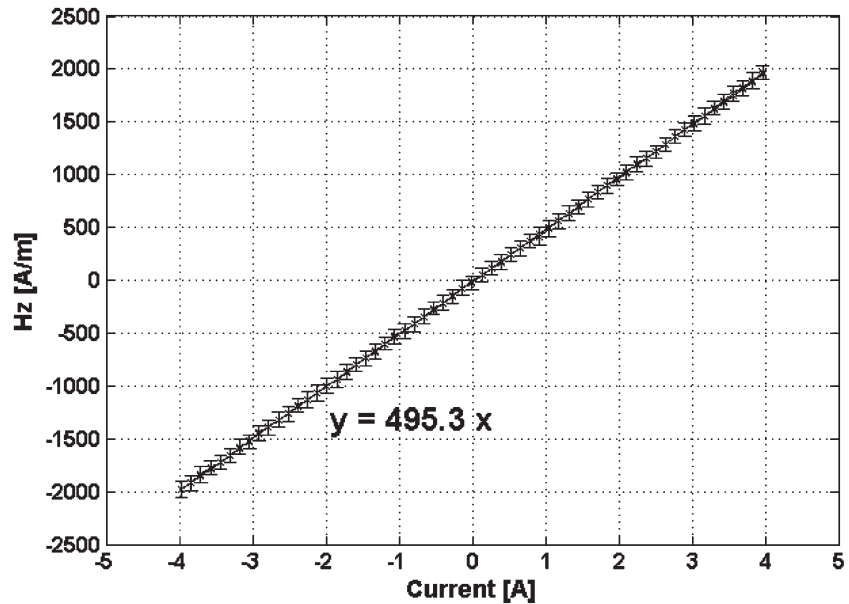

(a)

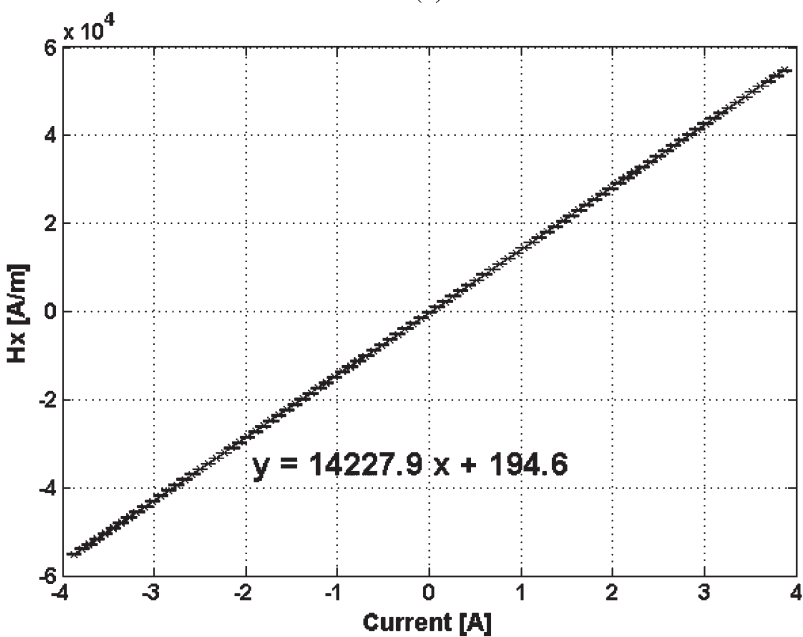

(b)

Fig. 5. (a) Solenoid and (b) resistive magnet $I-H$ transfer function

function $I-H$ to be used during the LVDT tests. The solenoid is a 33-cm-long plexiglas tube with a diameter of $3 \mathrm{~cm}$, on which 160 turns of a 1-mm-diameter wire are wound. The magnetic field $H$ was measured in air as a function of the input current $I$, in the range of $\pm 4 \mathrm{~A}$, at different positions along the tube length.

The $I-H$ curve with the uncertainty bar and the linear fit of the data are displayed in Fig. 5(a) and (b), for the solenoid and the resistive magnet, respectively. Both sources show good uniformity of the field along their length, being the measurements at different positions within the uncertainty bar of $\pm 3 \sigma$. The uncertainty, which was obtained by means of 50 repeated tests, resulted to be about $\pm 80 \mathrm{~A} / \mathrm{m}$, which is $0.1 \%$ and $0.01 \%$ of the maximum $H_{z}$ and $H_{x}$ values, respectively.

\section{EXPERIMENTAL RESULTS}

The potentiality of the test bench has been improved [12]. Two commercial LVDTs have been analyzed to prove the capability of the test bench to characterize the behavior of the sensor with and without an external magnetic field impinging on it.

\section{A. LVDT Characterization Without the External Field}

The sensors, which are called LVDT $A$ and LVDT B, have been analyzed without any external field by measuring their 


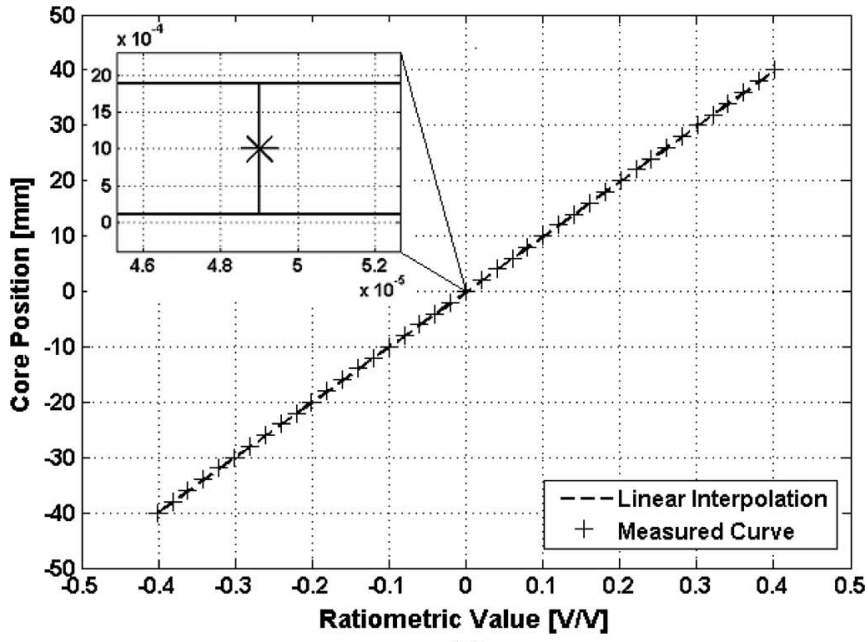

(a)

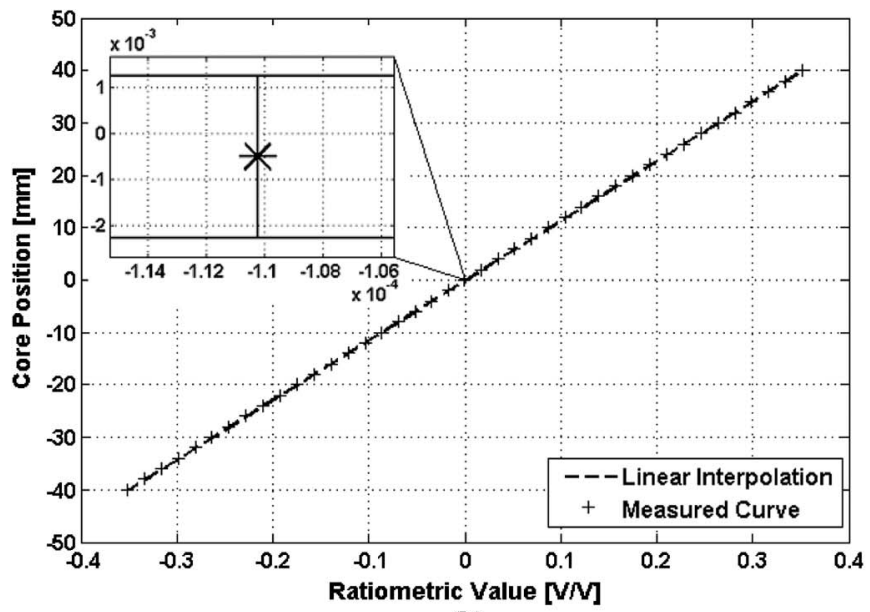

(b)

Fig. 6. Reading position as a function of (a) the ratiometric value for LVDT A and (b) the normalized differential value for LVDT B. Each point of the output characteristic was measured 50 times ( $a$ and b).

conversion tables $T$, between the array of position readings $p$ measured by the photoelectric encoder and assumed to be reference, and the corresponding ratiometric values $r$. Both sensors have a measurement range $(-P,+P)$ of $\pm 40 \mathrm{~mm}$. The ratiometric and normalized differential readings were used for LVDT A and LVDT B, respectively, as suggested by the manufacturers. In Fig. 6(a) and (b), the reading position is depicted as a function of the ratiometric (LVDT A) and of the normalized differential value (LVDT B), as previously mentioned. Uncertainty $u_{\mathrm{LVDT}+\text { read }}$ (type A) was evaluated by means of 50 repeated measurements.

The scan position $\pm 40 \mathrm{~mm}$ was carried out 30 times to assess the uncertainty (type A) $u_{p}$ of the positioning system, which turns out to be $\pm 2 \mu \mathrm{m}$. This uncertainty is due to the uncertainty values of the moving system (step motor) and the uncertainty of the feedback position sensor Heidenein. The composition of the uncertainty values $u_{p}$ and $u_{\mathrm{LVDT}+\text { read }}$ turns out to be the overall uncertainty $u$ of the test bench and of the LVDT under test when no magnetic interference is applied. The linearity and the uncertainty values $u_{\mathrm{LVDT}}+\mathrm{read}$ and $u_{p}$ are reported for the three main reading techniques of the LVDTs (see Table I).

\section{B. Discussion}

The best performance in terms of both linearity and uncertainty is achieved by using the reading techniques suggested by the manufacturers, i.e., the ratiometric reading for LVDT A and the normalized differential reading for LVDT B.

Generally, the techniques based on the ratiometric readings reduce the effects of the systematic errors of the acquisition system. As a matter of fact, the ratio operation allows reducing the gain errors of the acquisition system and mitigating temperature effects. The ratiometric technique also permits reducing the uncertainty of the reading value. By applying the uncertainty propagation law, it can be shown that the uncertainty of the ratiometric value $u_{r}$ is given by

$$
u_{r}^{2} \leq \frac{2\left(V_{\mathrm{sec} 1}^{2}+V_{\mathrm{sec} 2}^{2}\right)}{\left(V_{\mathrm{sec} 1}+V_{\mathrm{sec} 2}\right)^{4}}\left(u_{\mathrm{vsec} 1}^{2}+u_{\mathrm{vsec} 2}^{2}\right)
$$

where $u_{\mathrm{vsec} 1}$ and $u_{\mathrm{vsec} 2}$ are the uncertainty values (assumed equal) of $V_{\sec 1}$ and $V_{\sec 2}$, respectively, and $u_{d}$ is the uncertainty of the differential reading. Factor $k$ determines if uncertainty $u_{r}$ is minor than $u_{d}$. If the voltage signals are a few volts, as it is for LVDT A and LVDT B, $k$ is sensibly lower than 2, and $u_{r}$ will be lower than $u_{d}$. Similar considerations can be done for the ratiometric reading $\left(V_{\mathrm{sec} 1}-V_{\mathrm{sec} 2}\right) / V_{\mathrm{pri}}$.

However, one cannot directly draw any conclusion on the best reading technique, which basically depends on the LVDT design, its structure, and the coiling of the primary and secondary circuits. For example, it is interesting to note how the linearity of LVDT B drastically decreases if the ratiometric reading is used (see Table I). This is related to the design itself of the LVDT.

Nonlinearity NL (see Table I) represents the systematic error of the sensor and the acquisition system. The systematic errors of the acquisition system are corrected at each measurement by means of an autocalibration procedure. Due to the conversion table $T$, the systematic errors on the reading of the voltage amplitudes are taken into account by the calibration of the LVDT with respect to a reference position sensor. This is valid provided that the gain and offset of the board have good stability, although affected by a systematic error. Effectiveness of the autocalibration procedure is proven by the fact that the nonlinearity obtained by the differential reading is only slightly higher than that obtained by the reading technique suggested by the manufacturers (i.e., the ratiometric for LVDT A and the normalized differential for LVDT B), which mitigate the effect of systematic errors by definition since they are based on ratiometric operation. The nonlinearity of the sensor plays a major role and is due to the coiling of the primary and secondary circuits and the misalignment of the core.

The uncertainty of type-A $u_{\mathrm{LVDT}}+\mathrm{read}$, representing the uncertainty of the sensor itself and the reading system, resulted to be a few micrometers (see Table I). This performance is reached due to the high-sampling-rate and high-resolution analog-todigital converter (ADC) and by exploiting the sine-fit algorithm to evaluate the amplitudes of $V_{\mathrm{pri}}, V_{\mathrm{sec} 1}$, and $V_{\mathrm{sec} 2}$. The sinefit algorithm allows improving uncertainty on the measurement of the signal voltage amplitude since it reduces the effect of 
TABLE I

SUMmary DATA OF THE CALIBRATION FOR LVDTS A AND B

\begin{tabular}{|c|c|c|c|}
\hline \multicolumn{4}{|c|}{ LVDTA } \\
\hline Reading technique & $\left(\mathbf{V}_{\sec 1}-\mathbf{V}_{\sec 2}\right) /\left(\mathbf{V}_{\sec 1}+\mathbf{V}_{\sec 2}\right)$ & $\left(\mathbf{V}_{\text {sec1 }}-\mathbf{V}_{\text {sec } 2}\right) /\left(\mathbf{V}_{\text {pri }}\right)$ & $\left(\mathbf{V}_{\text {sec1 }}-\mathbf{V}_{\text {sec2 } 2}\right)$ \\
\hline Linearity (LVDT and reading system) & $0.07 \%$ & $0.09 \%$ & $0.12 \%$ \\
\hline$u_{L V D T+r e a d ~}( \pm 3 \sigma)$ & $\pm 1.0 \mu \mathrm{m}$ & $\pm 5.0 \mu \mathrm{m}$ & $\pm 4.8 \mu \mathrm{m}$ \\
\hline $\mathbf{u}( \pm \mathbf{3} \sigma)$ & $\pm 2.2 \mu \mathrm{m}$ & $\pm 5.4 \mu \mathrm{m}$ & $\pm 5.2 \mu \mathrm{m}$ \\
\hline \multicolumn{4}{|c|}{ LVDT B } \\
\hline Reading technique & $\left(\mathbf{V}_{\text {sec1 }}-\mathbf{V}_{\text {sec } 2}\right) /\left(\mathbf{V}_{\text {sec1 }}+\mathbf{V}_{\text {sec2 } 2}\right)$ & $\left(\mathbf{V}_{\text {sec1 }}-\mathbf{V}_{\text {sec } 2}\right) /\left(\mathbf{V}_{\text {pri }}\right)$ & $\left(\mathbf{V}_{\sec 1}-\mathbf{V}_{\sec 2}\right)$ \\
\hline Linearity (LVDT and reading system) & $15.2 \%$ & $0.1 \%$ & $0.2 \%$ \\
\hline $\mathbf{u}_{\text {LVDT+read }}( \pm 3 \sigma)$ & $\pm 6.7 \mu \mathrm{m}$ & $\pm 3.0 \mu \mathrm{m}$ & $\pm 3.3 \mu \mathrm{m}$ \\
\hline u $( \pm \mathbf{3} \sigma)$ & $\pm 7.0 \mu \mathrm{m}$ & $\pm 3.6 \mu \mathrm{m}$ & $\pm 3.9 \mu \mathrm{m}$ \\
\hline
\end{tabular}

random noise affecting the single ADC samples [3], [14]. If $N$ is the number of samples acquired, the uncertainty due to the random component is reduced roughly by factor $\sqrt{ }(N / 2)$. The full benefits of the sine-fit algorithm are discussed in [3], [14], and [15].

Finally, the results do not depend on the source type of the primary coil since the same figures were obtained using either a voltage or a current generator.

The overall uncertainty $u$ is dominated by uncertainty $u_{\mathrm{LVDT}+\text { read }}$ in all cases, except for LVDT A when the ratiometric technique is exploited. In the latter case, the overall uncertainty is dominated by uncertainty $u_{p}$ of the positioning system.

\section{LVDT Analysis With the External Magnetic Field}

The preceding results gave a figure of the overall uncertainty $u$ of the LVDT under test and the test bench (see Table I). This feature is crucial in order to correctly measure afterward the position drift $p_{i}-p_{i}^{\prime}$ due to an external magnetic field, which depends on the position itself. In fact, the uncertainty of the sensor, the reading system, and the positioning system have to be negligible than the measured position drift in order to prove that the latter is effectively due to the external interfering field.

The behavior of the sensor under a magnetic field will be assessed, and all of the three main reading techniques will be exploited without neglecting those that resulted to be less effective without any external magnetic interference. In fact, variations of the primary and secondary voltages in the presence of a magnetic field are not known a priori. Therefore, an experimental measurement is required to point out the best reading technique.

The position drift $p_{i}-p_{i}^{\prime}$, which is the difference between position $p_{i}$ read by the reference sensor and position $p_{i}^{\prime}$ read by the LVDT in the presence of a longitudinal magnetic field, was measured by applying the external interference $|H|$ up to a maximum intensity of about $800 \mathrm{~A} / \mathrm{m}$, i.e., a magnetic field that can be easily reached in an environment with motors and power cables [11], [12]. The test results are referred to external longitudinal interference since it causes a reading error that is about one order of magnitude larger than the horizontal one. The measurements were carried out for both LVDTs using voltage and current excitations. The three reading techniques,
TABLE II

Summary Data For the MAXIMUM POSITION DRIFT AT $15 \mathrm{~mm}$ IN THE CASE OF VOLTAGE AND CURRENT EXCITATIONS FOR LVDTS A AND B

\begin{tabular}{|c|c|c|}
\hline$\left(\mathbf{V}_{\mathrm{sec} 1}-\mathbf{V}_{\mathrm{sec} 2}\right) /\left(\mathbf{V}_{\mathrm{sec} 1}+\mathbf{V}_{\mathrm{sec} 1}\right)$ & $\left(\mathbf{V}_{\text {sec1 }}-\mathbf{V}_{\text {sec2 } 2}\right) /\left(\mathbf{V}_{\text {pri }}\right)$ & $\left(\mathbf{V}_{\mathrm{sec} 1}-\mathbf{V}_{\mathrm{sec} 2}\right)$ \\
\hline \multicolumn{3}{|c|}{ LVDT A: Voltage excitation } \\
\hline $180 \mu \mathrm{m}$ & $270 \mu \mathrm{m}$ & $310 \mu \mathrm{m}$ \\
\hline \multicolumn{3}{|c|}{ LVDT A: Current excitation } \\
\hline $200 \mu \mathrm{m}$ & $290 \mu \mathrm{m}$ & $3500 \mu \mathrm{m}$ \\
\hline \multicolumn{3}{|c|}{ LVDT B: Voltage excitation } \\
\hline $410 \mu \mathrm{m}$ & $160 \mu \mathrm{m}$ & $170 \mu \mathrm{m}$ \\
\hline \multicolumn{3}{|c|}{ LVDT B: Current excitation } \\
\hline $420 \mu \mathrm{m}$ & $171 \mu \mathrm{m}$ & $555 \mu \mathrm{m}$ \\
\hline
\end{tabular}

i.e., ratiometric $\left(\left(V_{\mathrm{sec} 1}-V_{\mathrm{sec} 2}\right) /\left(V_{\mathrm{sec} 1}+V_{\mathrm{sec} 2}\right)\right)$, differential $\left(V_{\mathrm{sec} 1}-V_{\mathrm{sec} 2}\right)$, and differential normalized readings to the primary voltage $\left(\left(V_{\mathrm{sec} 1}-V_{\mathrm{sec} 2}\right) /\left(V_{\mathrm{pri}}\right)\right)$, were used to evaluate the position on the basis of the coil voltage in order to study how the drift changes. In particular, the drifts at three nominal positions, i.e., \pm 15 and $0 \mathrm{~mm}$, due to increasing $H \uparrow$ and decreasing $(H \downarrow)$ longitudinal magnetic fields, are reported for current and voltage excitations in Figs. 7 (LVDT A) and 8 (LVDT B). In this case, the ratiometric and normalized differential readings have been used for LVDTs A and B, respectively. The magnetic cycle has been repeated 30 times for each position, and the average value of the position drift with a $\pm 3-\sigma$ uncertainty bar is reported (see vertical bars of Figs. 7 and 8).

The position drift at the maximum interfering field of $800 \mathrm{~A} / \mathrm{m}$ is reported for both excitation types (voltage and current) and both LVDTs in Fig. 9. The drift is referred at the position of $15 \mathrm{~mm}$, where the drift gets worse (see Figs. 7 and 8). The results of different reading techniques have been investigated. The maximum position drifts are summarized in Table II for both LVDTs. Data refer to the position of $15 \mathrm{~mm}$ and the external magnetic field of $800 \mathrm{~A} / \mathrm{m}$.

\section{Discussion}

As shown in Figs. 7-9, the position drift $p_{i}-p_{i}^{\prime}$ results to be hundreds of micrometers, which is a factor of 100 higher than the sensors' uncertainty for both LVDTs (see Tables I and II). Therefore, it is evident that an external magnetic field drastically affects the LVDT's measurement performance.

As a further result, the highlights in Figs. 7 and 8 show the 3- $\sigma$ uncertainty bar of the position drift, which resulted to be 


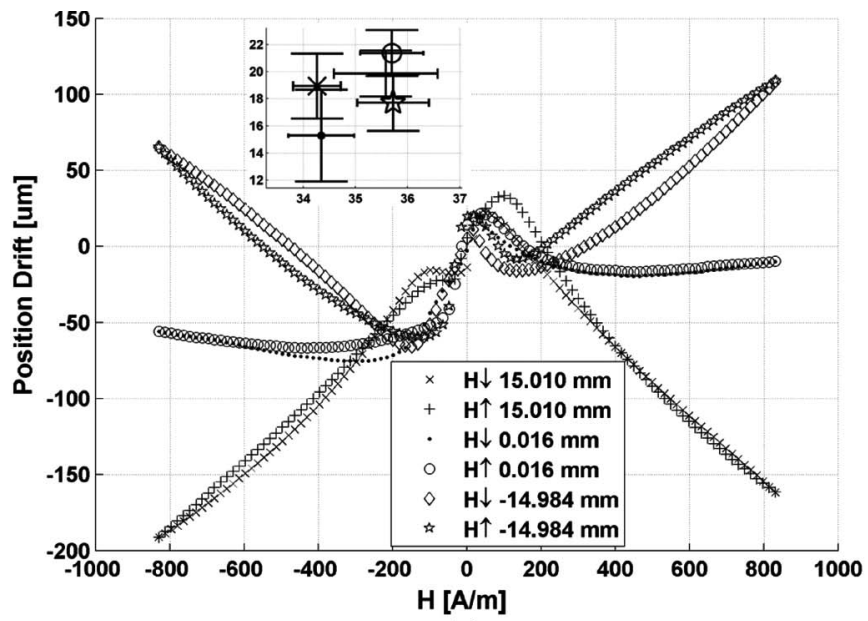

(a)

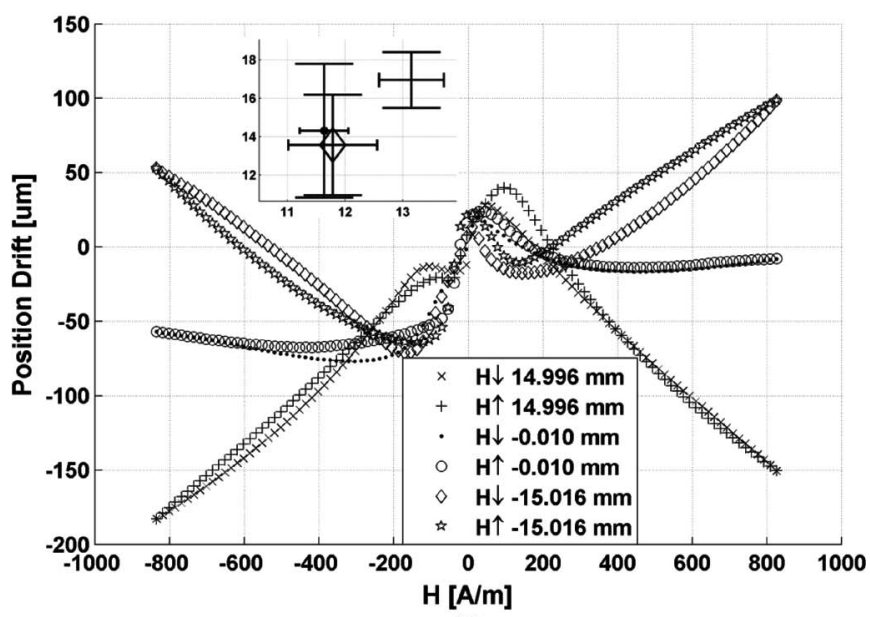

(b)

Fig. 7. Position drift due to a longitudinal interference of $800 \mathrm{~A} / \mathrm{m}$ for LVDT $\mathrm{A}$ in the case of (a) current and (b) voltage excitations. The highlight shows the $3-\sigma$ uncertainty bar (30 repeated measurements) on both axes at null position.

only a few micrometers for both LVDTs A and B. This proves that the test bench assures good measurement repeatability also in the presence of the external magnetic field.

LVDTs A and B exhibit a different behavior when a longitudinal magnetic field impinges on them. First, one can note that the hysteresis cycle of LVDT B is larger than the one exhibited by LVDT A (see Figs. 7 and 8). This is mainly due to the difference of the materials, which the LVDTs are made of. Then, the position drift for LVDT B presents a kind of polarization that increases according to the displacement (see Fig. 8), whereas the drift of LVDT A is centered at about the null value. Those different behaviors could be read as a consequence of the difference in the hysteresis cycles exhibited by the LVDTs.

Despite of this difference, the performance of LVDTs A and B can be comparable if one chooses the most suitable supply excitation and reading technique to increase the robustness of the sensor with respect to an external magnetic field. In fact, the maximum drift (at $15 \mathrm{~mm}$ and $800 \mathrm{~A} / \mathrm{m}$ ) can be reduced at ca. 160-180 $\mu \mathrm{m}$ if the sensors are supplied by a voltage source and read by exploiting the ratiometric and normalized differential techniques for LVDTs A and B, respectively.

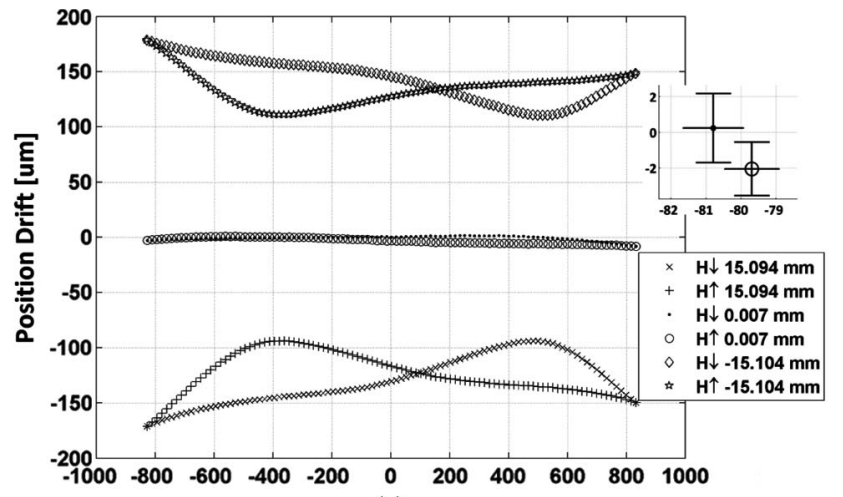

(a)

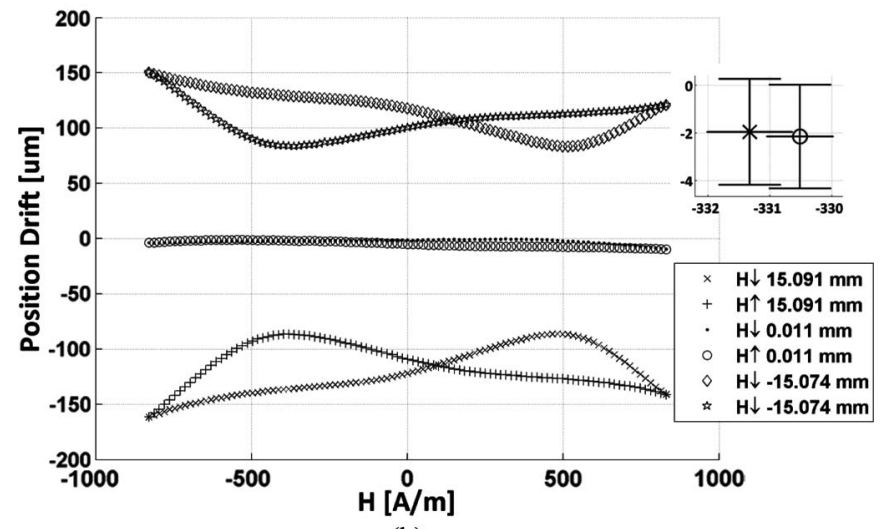

(b)

Fig. 8. Position drift error due to a longitudinal interference of $800 \mathrm{~A} / \mathrm{m}$ for LVDT B in the case of (a) current and (b) voltage excitations. The highlight shows the 3- $\sigma$ uncertainty bar (30 repeated measurements) on both axes at null position.

The current excitation also gives good results for LVDT B (see Table II).

In more details, as far as LVDT A is concerned, the position drift does not depend on the source excitation (see Fig. 7) when the ratiometric reading technique is used. The same consideration applies for the normalized differential reading (see Fig. 9 and Table II). The differential reading gives the worst results and is not recommended in the case of a current excitation for which the position drift increases at the order of millimeters.

As far as LVDT B is concerned, the position drift does not depend on the source excitation when the normalized differential reading technique or the ratiometric one is applied (see Fig. 8). However, the former, which is the suggested technique of the manufacturer, gives better results than the latter, contrarily to the case of LVDT A (see Fig. 9 and Table II). The differential technique gives good results in the case of a voltage excitation but gets much worse in the case of a current supply (see Table II).

As a matter of fact, an external field affects the impedance of the primary and, therefore, the primary voltage in the case of a current excitation. That variation is not taken into account in the differential reading, which turns out to be the worst case for a current supply. The variation of the primary voltage is less important in the case of a voltage excitation. Further details on the phenomenon model are given in [16]. 


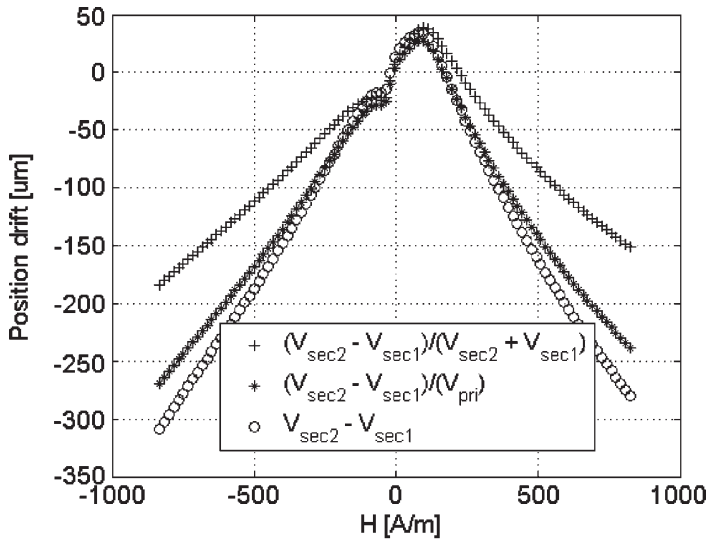

(a)

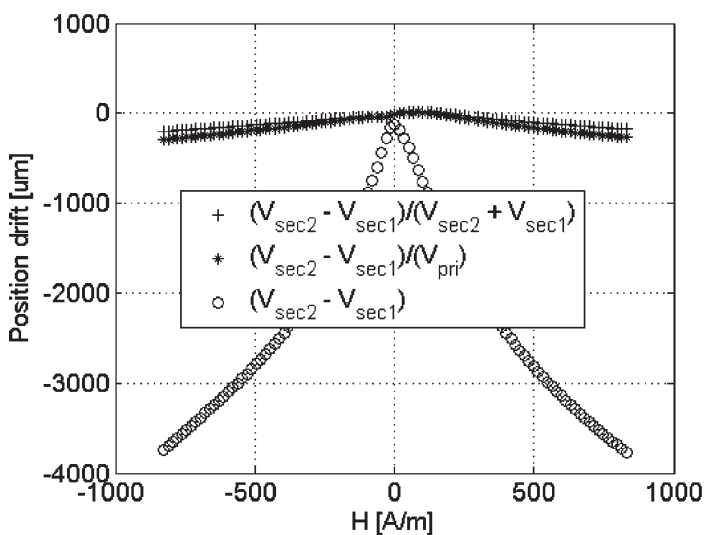

(b)

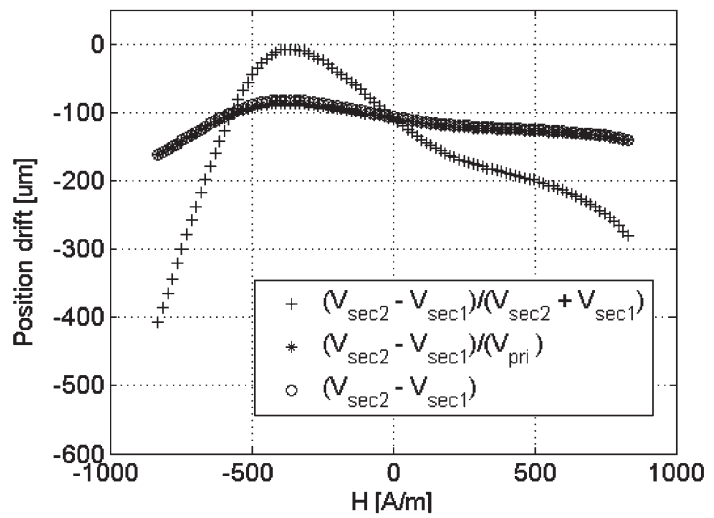

(c)

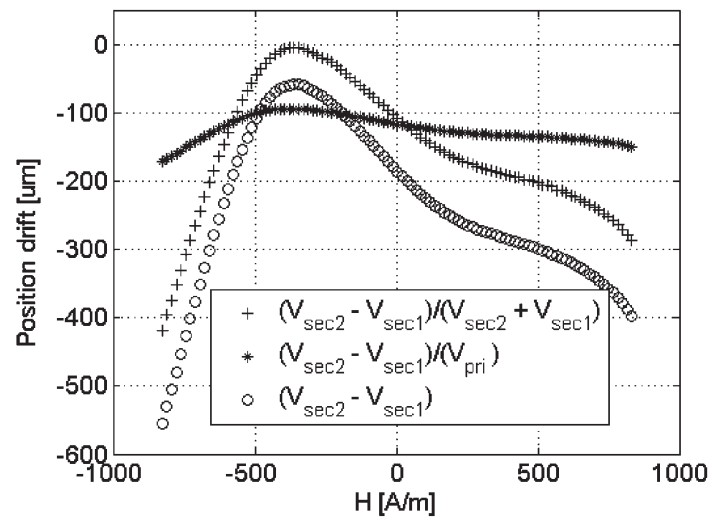

(d)

Fig. 9. Position drift at $15 \mathrm{~mm}$ due to a longitudinal interference of $800 \mathrm{~A} / \mathrm{m}$. Different reading techniques are compared. (a) Voltage and (b) current excitations of LVDT A. (c) Voltage and (d) current excitations of LVDT B.

\section{Conclusion}

A detailed procedure and a test bench for measuring LVDT performance in the presence of interfering magnetic fields impinging on it have been presented.

The procedure allows evaluating the sensor characteristics such as the nonlinearity and the uncertainty with and without an external magnetic field. The position drift of the sensor due to the effect of magnetic fields has been assessed. Those tests can be carried out by using a current or a voltage source, and the position can be evaluated according to different reading techniques.

Two commercial LVDTs have been tested. A maximum position drift of hundreds of micrometers has been measured on both sensors, proving that a magnetic interference can deeply affect their precision, which is normally on the order of a few micrometers. The curves of the position drift are not obvious to understand. In fact, the influence of an external magnetic field depends on the magnetic properties of the sensor material, mainly the core and the foil.

The measurement and the test bench have proved to be valuable tools to study the effects of interfering fields on the sensors in order to investigate the most suitable excitation source and reading technique for the specific sensor under test.

Finally, the repeated tests have showed that the overall uncertainty of the test bench is a few micrometers. This feature has also been confirmed when applying the measurement procedure to evaluate the effect of an external magnetic field on the reading position of an LVDT sensor.

\section{ACKNOWLEDGMENT}

The authors would like to thank Prof. V. G. Vaccaro, Prof. F. Cennamo, and Prof. P. Arpaia for their useful discussions and suggestions and A. Danisi for his collaboration in the analysis of the last measurement results.

\section{REFERENCES}

[1] M. Martino, A. Danisi, R. Losito, A. Masi, and G. Spiezia, "Design of a LVDT with high rejection to external interfering magnetic field," IEEE Trans. Magn., vol. 46, no. 2, pp. 674-677, Feb. 2010.

[2] K. Ara, "A differential transformer with temperature- and excitationindependent output," IEEE Trans. Instrum. Meas., vol. IM-21, no. 3, pp. 249-255, Aug. 1972.

[3] A. Masi, A. Brielmann, R. Losito, and M. Martino, "LVDT conditioning on the LHC collimators," IEEE Trans. Nucl. Sci., vol. 55, no. 1, pp. 67-75, Feb. 2008.

[4] A. Masi and R. Losito, "LHC collimators low level control system," IEEE Trans. Nucl. Sci., vol. 55, no. 1, pp. 333-340, Feb. 2008.

[5] R. Rajamani and J. Hedrick, "Adaptive observers for active automotive suspension: Theory and experiment," IEEE Trans. Control Syst. Technol., vol. 3, no. 1, pp. 86-93, Mar. 1995.

[6] D. Buehler, T. R. Oxland, and L.-P. Nolte, "Design and evaluation of a device for measuring three-dimensional micromotions of a press-fit femoral stem prostheses," Med. Eng. Phys., vol. 19, no. 2, pp. 187-199, Mar. 1997.

[7] R. S. Weissbach, D. R. Loker, and R. M. Ford, "Test and comparison of LVDT signal conditioner performance," in Proc. 17th IEEE Instrum. Meas. Technol. Conf., 2000, pp. 1143-1146.

[8] F. Yassa and S. Garverick, "A multicahnnel digital demodulator for LVDT/RVDT position sensor," IEEE J. Solid-State Circuits, vol. 25, no. 2, pp. 441-450, Apr. 1990.

[9] G. Novacek, "Accurate linear measurement using LVDTs," Circuit Cellar Ink, no. 106, pp. 20-27, May 1999. 
[10] D. Crescini, A. Flammini, D. Marioli, and A. Taroni, "Application of an FFT-based algorithm to signal processing of LVDT position sensors," IEEE Trans. Instrum. Meas., vol. 47, no. 5, pp. 1119-1123, Oct. 1998.

[11] A. Danisi, "Simulation of DC interfering magnetic field effects on the LHC collimators' LVDT positioning sensors," M.S. thesis, Dept. Electron. Eng., Univ. "Federico II", Naples, Italy, 2009.

[12] G. Spiezia, R. Losito, M. Martino, A. Masi, and A. Pierno, "Automatic test bench for the measurement of the magnetic interference on LVDTs," in Proc. I2MTC, Austin, TX, May 2010, pp. 850-854.

[13] D. S. Nyce, "The LVDT," in Linear Position Sensors. Hoboken, NJ: Wiley-Interscience, 2004, pp. 100-101.

[14] F. Corrêa Alegria, "Bias of amplitude estimation using three-parameter sine fitting in the presence of additive noise," Measurement, vol. 42, no. 5, pp. 748-756, Jun. 2009.

[15] M. Martino, R. Losito, and A. Masi, "Analytical metrological characterization of the three-parameter sine fit algorithm," unpublished.

[16] M. Martino, G. Golluccio, R. Losito, and A. Masi, "An analytical model of the effect of external DC magnetic fields on the AC voltages of an LVDT," in Proc. IEEE Int. Instrum. Meas. Technol. Conf., Austin, TX, May 3-6, 2010, pp. 213-218.

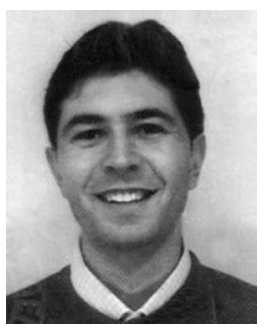

Giovanni Spiezia was born in Naples, Italy, on January 1981. He received the M.D. degree in electronic engineering and the Ph.D. degree in measurement systems from the University of Naples Federico II, Naples. His Ph.D. activity was carried out with the European Organization for Nuclear Research (CERN), Geneva, Switzerland, and was focused on the design and development of a fast digital integrator for measuring dynamic properties of superconducting magnets.

$\mathrm{He}$ is currently with the Engineering Department, CERN, where he is involved in the development of a new position sensor prototype, design of new radiation sensors to evaluate the impact of nuclear radiation on electronics, and radiation testing of electronic devices. His research interests include electronic design, digital signal processing, and testing of radiation tolerant electronics.

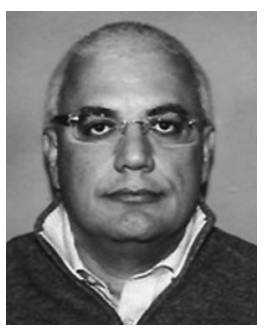

Roberto Losito was born in Naples, Italy. He received the M.D. degree in electronic engineering from the University of Naples Federico II, Naples, in 1992.

In 1994, he was a Fellow with the Laboratoire de l'Accélérateur Linéaire, Orsay, France, and with the Institute of Research for Electromagnetics and Electronic Components-National Research Council, Naples. Since 1995, he has been a Staff of the Engineering Department, European Organization for Nuclear Research, Geneva, Switzerland. He participated in the design and construction of radio-frequency systems for the Large Hadron Collider (LHC) for both normal conducting and superconducting systems. Then, he led a group in charge of design of a LHC collimator low-level control system and a team in charge of compact linear collider photoinjectors. Since 2008, he has been in charge of a group responsible for targets, radioactive ion and electron sources, and studies on radiation tolerance of control electronics. His research interests include radio frequency, control systems, and particle sources.

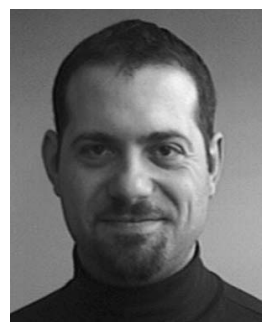

Michele Martino was born in Naples, Italy, on July 23,1975 . He received the M.D. degree in electronic engineering from the University of Naples Federico II, Naples, where he is currently working toward the Ph.D. degree in electrical engineering. $\mathrm{His} \mathrm{Ph} . \mathrm{D}$. research on the high-precision remote control and position survey of Large Hadron Collider collimators is carried out with the Sources, Targets and Interactions Group, Engineering Department, European Organization for Nuclear Research (CERN), Geneva, Switzerland.

In 2004, he was a Control Algorithm Designer on traction control with Fiat Group Automobiles. In 2005, he was with the Italian National Institute of Nuclear Physics (INFN) as a Fellow and worked on LHCb muon detector frontend electronic test and characterization. Since June 2006, he has been with CERN, first as a Project Associate and, then, as a Doctoral Student. He recently joined the Electrical Power Converter Group to work on electrical precision measurement required by power converters of CERN accelerators. His research interests include linear variable differential transformer sensor characterization and conditioning, sensorless control of a stepping motor driven by very long cables, and high-precision pulsed current measurement.

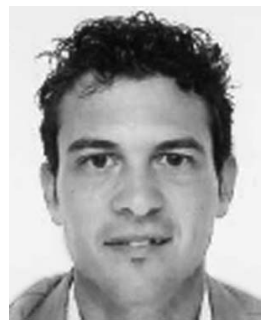

Alessandro Masi was born in Naples, Italy, on July 19, 1976. He received the M.D. degree in electronic engineering and the Ph.D. degree in computer science from the University of Naples Federico II, Naples. His Ph.D. research on high-accuracy measurement systems for superconducting magnets of the new particle accelerator Large Hadron Collider was carried out with the Magnetic Measurements and Tests Group, European Organization for Nuclear Research (CERN), Geneva, Switzerland.

$\mathrm{He}$ has been a Researcher with the InterUniversities Consortium of Computer Science (CINI) on network architecture for remote measurement and control systems. Then, he was with STMicroelectronics as a Digital Designer for one year. Since April 2005, he has been a CERN Staff Member in the Sources, Targets and Interactions Group. Since January 2008, he leads the Equipment Controls and Electronics Section. The section is responsible for the design, installation, and maintenance of control systems on different platforms (e.g., programmable logic controllers, peripheral component interconnect extensions for instrumentation, and virtual machine environment) for all equipment under the group's responsibility, mainly movable devices characterized by few micrometer positioning accuracy (e.g., scrapers, collimators, shielding, and targets) in a hard radioactive environment. Important section activities are the development of radiation-hard motors and sensors, precision electronics compatible with long cables, and high immunity to electromagnetic interference.

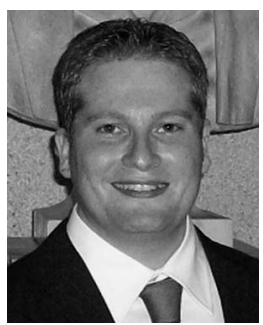

Antonio Pierno was born in Naples, Italy, on May 1981. He received the M.D. degree in electronic engineering, in 2009, from the University of Naples Federico II, Naples, where he is currently working toward the Ph.D. degree with the Department of Electrical Engineering, University of Naples. He carried out his final thesis work with the European Organization for Nuclear Research (CERN), Geneva, Switzerland, by developing an automatic test bench for characterization of magnetic interference on linear variable differential transformers (LVDTs).

From January 2009 to February 2010, he was with the Sources, Targets and Interactions Group, Equipment Controls and Electronics Section, Engineering Department, CERN, where he was engaged in the study of phenomena regarding LVDTs and their behavior in the presence of an external magnetic field. His research interests include transients in power systems, lightning effects on power lines, and power quality problems. 